\title{
Protein Tyrosine Phosphatase N1 Gene Variants Associated with Type 2 Diabetes Mellitus and Its Related Phenotypes in the Korean Population
}

\author{
Kyung-Won Hong', Hyun-Seok $\mathrm{Jin}^{1}$, Ji-Eun \\ $\mathrm{Lim}^{1}$, Ha-Jung $\mathrm{Ryu}^{2}$, Younjhin Ahn', Jong- \\ Young Lee ${ }^{2}$, Bok Ghee Han', Hyoung Doo \\ $\mathrm{Shin}^{3,4}$, Nam Han $\mathrm{Cho}^{5}$, Chol Shin ${ }^{6}$, Jeong- \\ Taek Woo ${ }^{7}$, Hun Kuk Park ${ }^{1}$ and Bermseok \\ $\mathrm{Oh}^{1 *}$
}

${ }^{1}$ Biomedical Education Center, Department of Biomedical Engineering, School of Medicine, KyungHee University, Seoul 130-701, Korea, ${ }^{2}$ Center for Genome Science, National Institute of Health, Seoul 122-701, Korea, ${ }^{3}$ SNP Genetics, Inc., Seoul 153-803, Korea, ${ }^{4}$ Department of Life Science, Sogang University, Seoul 121-742, Korea, ${ }^{5}$ Department of Preventive Medicine, Ajou University School of Medicine, Suwon 443-749, Korea, ${ }^{6}$ Department of Internal Medicine, Korea University Ansan Hospital, Gyeonggi-do 425-707, Korea, ${ }^{7} \mathrm{De}-$ partment of Endocrinology and Metabolism, KyungHee University Hospital, School of Medicine, KyungHee University, Seoul 130-701, Korea

\begin{abstract}
Protein phosphorylation at tyrosine residues is a key regulatory event that modulates insulin signal transduction. We studied the PTPN1 gene with regard to susceptibility to Korean type 2 diabetes mellitus (T2DM) and its related quantitative traits. $A$ total of seven SNPs [g.36171G $>$ A (rs941798), g.58166G $>A \quad$ (rs3787343), g.58208A $>G \quad($ rs2909270), g.64840C $>T \quad($ rs754118), g.69560C $>G$ (rs6020612), g.69866G $>A$ (rs718050), and g.69934T $>$ G (rs3787343)] were selected based on frequency $(>0.05)$, linkage disequilibrium (LD) status, and haplotype tagging status. We studied the seven SNPs in 483 unrelated patients with type 2 diabetes (age: $64 \pm 2.8$ years, onset age: $56 \pm 8.1$ years; 206 men, 277 women) and 1138 nondiabetic control subjects (age: $64 \pm 2.9 ; 516$ men, 622 women). The SNP rs941798 had protective effects against T2DM with an odds ratio of 0.726 (C.I. $0.541 \sim 0.975$ ) and $p$-value $=0.034$, but none of the remaining six SNPs was associated with T2DM. Also, rs941798 was associated with blood pressure,
\end{abstract}

*Corresponding author: E-mail ohbs@khu.ac.kr Tel +82-2-961-0290, Fax +82-2-961-5515

Accepted 2 September 2008
HDL cholesterol, insulin sensitivity. rs941798 also has been associated with T2DM in previous reports of Caucasian-American and Hispanic-American populations. This is the first report that shows an association between PTPN1 and T2DM in the Korean as well as Asian population.

Keywords: PTPN1, insulin signaling, Korean, T2DM, rs941798

\section{Introduction}

Protein phosphorylation at tyrosine residues modulates intracellular signaling pathways, and this modulation is an essential determinant of insulin signal transduction (Goldstein et al., 1998; Evans and Jallal, 1999). Protein tyrosine phosphatase 1B protein (PTP1B), encoded by the PTPN1 gene, regulates the tyrosine phosphorylation of insulin receptor (Seely et al., 1996) and insulin receptor substrate 1 (Goldstein et al., 2000), which leads to downregulation of insulin signaling. PTPN1 deficiency in mice results in increased insulin sensitivity (Elchebly et al., 1999) and enables normalization of blood glucose levels (Klaman et al., 2000). Moreover, it has been shown that inactivation with antisense oligonucleotides regulates the expression of genes that are involved in lipogenesis, such as SREBF1, suggesting that PTP1B may play a role in the enlargement of adipocyte energy storage (Rondinone et al., 2002).

The human PTPN1 gene maps to chromosome $20 q 13.13$, a syntenic region of the distal arm of mouse chromosome 2 that harbors quantitative trait loci for body fat and body weight (Lembertas et al., 1997). The PTPB1 gene consists of 10 exons, spanning $74 \mathrm{~kb}$, and the first intron is longer than $50 \mathrm{~kb}$. In humans, several linkage signals with type 2 diabetes mellitus (T2DM) (Bowden et al., 1997), BMl (Hunt et al., 2001), fat mass, and energetic intake (Collaku et al., 2004; Dong et al., 2003; Lembertas et al., 1997) were reported at this locus in different populations, further supporting the candidacy of PTPN1 involvement in T2DM and obesity. In Poland, a family-based linkage study of T2DM showed the highest logarithm of the odds score (Ji et al., 1997; Klupa et al., 2000). This locus also showed evidence of linkage with early onset T2DM (onset=45 years) in a subset of 55 French families (Zouali et al., 1997). 
Since the discovery of the PTPN1 gene (Forsell et al., 2000), multiple studies have attempted to examine its role in susceptibility to T2DM. A missense variant in the coding region of PTPN1 was found to be associated with T2DM and glucose tolerance in the Danish population (Echwald et al., 2002), and an insertion/deletion polymorphism in the 3'-untranslated region was associated with insulin resistance (Di Paola et al., 2002). In an extensive analysis of the PTPN1 gene locus, Bento et al. (2004) observed associations between multiple SNPs and T2DM in two independent CaucasianAmerican case-control samples. The evidence for association was most consistent for SNPs in the region that spanned the 3'-end of intron 1 to the last, intron 8 . All of the associated SNPs lay in a single haplotype block, and one common haplotype (frequency $=36 \%$ ) was found to be strongly associated with T2DM. The same group evaluated and confirmed previous findings of SNPs and haplotypes of PTPN1 for association with quantitative glycemic traits in Hispanic-American subjects from the Insulin Resistance Atherosclerosis Study Family Study (IRASFS) (Palmer et al., 2004). However, a recent meta-analysis that included 7883 individuals from three large European case-control samples (from the US, Poland, and Scandinavia) did not replicate this association for any single SNP or haplotype (Florez et al., 2005). A conclusion of the association between PTPN1 and T2DM in Asian and African populations is needed.

Despite the inconsistency of the association with T2DM, the evidence that PTPN1 might be a significant contributor to genetic susceptibility to T2DM seems to be strong. Because no study of PTPN1 in association with T2DM in the Asian population has been presented, we provide the first report of the susceptibility of the PTPN1 gene in Korean T2DM and its related quantitative traits.

\section{Methods}

\section{Subjects}

The 24 DNA samples from Korean subjects that were used for the initial sequencing were randomly selected from unrelated local residents with no history of familial diseases. With 24 samples (48 chromosomes), it might be expected that more than $90 \%$ of SNPs have a frequency that is greater than 0.05 (Eberle and Kruglyak, 2000). We studied 483 unrelated patients with type 2 diabetes (age: $64 \pm 2.8$ years, onset age: $56 \pm 8.1$ years; 206 men, 277 women) and 1138 nondiabetic control subjects (age: $64 \pm 2.9 ; 516$ men, 622 women). All subjects who enrolled in this study originated from the Ansung-Ansan prospective community cohort.
In this study, diabetic subjects were recruited according to American Diabetes Association criteria from 10,038 individuals in the Ansung-Ansan prospective community cohort. Nondiabetic subjects who had no history of diabetes, no first-degree relatives with diabetes, fasting plasma glucose levels less than 6.1 $\mathrm{mmol} / \mathrm{L}$, and hemoglobin $\mathrm{A} 1 \mathrm{c}(\mathrm{HbA} 1 \mathrm{c})$ levels less than $5.8 \%$ were recruited from the cohort. To be eligible, normal control subjects also had to be free of medications for diabetes, hypertension, and dyslipidemia. The study was approved by the institutional review board of the Korean National Institute of Health. Written informed consent was obtained from all subjects.

\section{Measurement of clinical characteristics}

Both T2DM and normal control subjects were aged 60 years and older. The clinical characteristics of the subjects are summarized in Table 1. All study subjects were examined in the morning after an overnight fast and 60 min and $120 \mathrm{~min}$ after an oral glucose tolerance test (OGTT). The parameters that were measured were height, weight, body mass index [BMl: weight $(\mathrm{kg}) /$ square of height $\left.\left(\mathrm{m}^{2}\right)\right]$, waist and hip circumference, waist-to-hip ratio (WHR: waist/hip), and blood pressure. Blood samples were drawn for biochemical measurements: fasting plasma glucose (GLU0), OGTT $60 \mathrm{~min}$ and 120 min plasma glucose (GLU60 and GLU120, respectively), fasting plasma insulin (INSO), OGTT $60 \mathrm{~min}$ and 120 min plasma insulin (INS60 and INS120, respectively), $\mathrm{HbA1c}$, total cholesterol (TCHL), triglycerides (TG), and high-density lipoprotein cholesterol (HDL). Using the measurements, low-density lipoprotein cholesterol [LDL: TCHL-TG-(HDL/5)], area under glucose curve (AUCGLU), area under insulin curve (AUCINS), homeostasis model assessment of insulin resistance [HOMAIR: GLUO*INS0/(22.5*14.182)], quantitative insulin sensitivity check index [QUICKI: 1/(log(GLUO)+ log(INSO)], and insulin sensitivity index (ISI) were calculated. The QUICKI was derived using the inverse of the sum of the logarithms of the fasting insulin and fasting glucose levels. This index correlates well with glucose clamp studies ( $r=0.78)$ and is useful for measuring insulin sensitivity (IS), which is the inverse of insulin resistance (IR) (Katz et al., 2000).

\section{Sequencing analysis of the human PTPN1 gene}

We sequenced all exons, including exon-intron boundaries and the promoter region (approximately $1.5 \mathrm{~kb}$ ), to discover single nucleotide polymorphisms (SNPs) in 24 DNA samples from Koreans using the ABI PRISM 3730 DNA analyzer (Applied Biosystems, Foster City, CA, 
USA). Sixteen primer sets for the amplification and sequencing analysis were designed based on GenBank sequences (Ref. Genome seq. NT_011362 released on February 19, 2004). Information regarding primers is available on our website (http://www.ngri,re.kr/SNP/). Sequence variants were verified by chromatograms.

\section{Genotyping}

Among the identified polymorphisms, seven SNPs (g.36171G $>$ A, g.58166G $>A, 58208 A>G, g .64840 C>T$, g.69560C $>$ G, g.69866G $>A$, and g.69934T $>G$ ) were selected based on frequency $(>0.05)$, linkage disequilibrium (LD) status, and haplotype tagging status. In addition, one indel (g.1484G_ins) was selected based on previous reports (Bento et al., 2004; Burdon et al., 2006).

SNPs were genotyped using amplifying primers and probes designed for TaqMan (Livak, 1999). Primer Express (Applied Biosystems) was used to design both the PCR primers and the MGB TaqMan probes. One allelic probe was labeled with the FAM dye, and the other was labeled with the fluorescent VIC dye. PCRs were run in TaqMan Universal Master mix without UNG (Applied Biosystems) and with PCR primer concentrations of $900 \mathrm{nM}$ and TaqMan MGB probe at a concentration of $200 \mathrm{nM}$. Reactions were performed in 384-well format in a total reaction volume of $5 \mu \mathrm{l}$ using $20 \mathrm{ng}$ of genomic DNA. The plates were then placed in a thermal cycler (PE 9700, Applied Biosystems) and heated at $50^{\circ} \mathrm{C}$ for $2 \mathrm{~min}$ and $95^{\circ} \mathrm{C}$ for $10 \mathrm{~min}$, followed by 40 cycles of $95^{\circ} \mathrm{C}$ for $15 \mathrm{~s}$ and $60^{\circ} \mathrm{C}$ for $1 \mathrm{~min}$, with a final soak at $25^{\circ} \mathrm{C}$. The TaqMan assay plates were transferred from the thermal cyclers to a real-time PCR system (Prism 7900HT, Applied Biosystems) that read the fluorescence intensity of each well of the plate. Fluorescence data files from each plate were analyzed using automated software (SDS ver. 2.1, Applied Biosystems).

\section{Statistics}

Differences in anthropometric and physiologic variables between nondiabetic and diabetic subjects were com-

Table 1. Clinical characteristics of study subjects

\begin{tabular}{|c|c|c|c|c|c|c|c|c|c|}
\hline \multirow{2}{*}{ Variables } & \multirow{2}{*}{ Abbreviation } & \multicolumn{3}{|c|}{$\begin{array}{l}\text { Normal Control } \\
\qquad(n=1,138)\end{array}$} & \multicolumn{3}{|c|}{ T2DM $(n=483)$} & \multirow{2}{*}{$\begin{array}{c}\text { Test } \\
\text { statistic* }^{*}\end{array}$} & \multirow{2}{*}{$\mathrm{p}$-value } \\
\hline & & $N$ & Mean & $S D$ & $N$ & Mean & $S D$ & & \\
\hline Age (yrs) & & 1,138 & 64.237 & 2.873 & 483 & 64.621 & 2.779 & -2.480 & 0.0130 \\
\hline Onset (yrs) & & & & & 271 & 56.288 & 8.091 & & \\
\hline Duration (mths) & & & & & 271 & 101.181 & 94.071 & & \\
\hline Sex (men/women) & & 516 & $5.34) / 622$ & $(54.66)$ & $206(4$ & $2.65) / 277$ & $7(57.35)$ & 0.995 & 0.3190 \\
\hline Body mass index $\left(\mathrm{Kg} / \mathrm{m}^{2}\right)$ & BMI & 1,138 & 23.660 & 3.143 & 483 & 25.128 & 3.154 & -8.590 & $<.0001$ \\
\hline Waist-to-hip ratio & WHR & 1,137 & 0.913 & 0.066 & 482 & 0.932 & 0.067 & -5.190 & $<.0001$ \\
\hline Systolic blood pressure (mm Hg) & SBP & 1,138 & 124.773 & 18.580 & 483 & 129.170 & 18.326 & -4.380 & $<.0001$ \\
\hline Diastolic blood pressure $(\mathrm{mm} \mathrm{Hg})$ & DBP & 1,138 & 77.418 & 10.158 & 483 & 77.600 & 10.357 & -0.330 & 0.7433 \\
\hline Triglyceride $(\mathrm{mg} / \mathrm{dL})$ & $\mathrm{TG}$ & 1,138 & 147.679 & 72.202 & 483 & 199.887 & 140.273 & -7.760 & $<.0001$ \\
\hline Total cholesterol (mg/dL) & $\mathrm{TCHL}$ & 1,138 & 185.431 & 33.947 & 483 & 194.884 & 42.264 & -4.360 & $<.0001$ \\
\hline High-density lipoprotein cholesterol (mg/dL) & HDL & 1,138 & 45.208 & 10.193 & 483 & 42.678 & 9.857 & 4.610 & $<.0001$ \\
\hline Low-density lipoprotein cholesterol (mg/dL) & LDL & 1,125 & 111.080 & 31.568 & 452 & 115.586 & 38.382 & -2.210 & 0.0270 \\
\hline Fasting plasma glucose (mg/dL) & GLU0 & 1,138 & 80.625 & 7.755 & 326 & 118.028 & 35.242 & -19.030 & $<.0001$ \\
\hline $\begin{array}{l}\text { Plasma glucose after } 60 \mathrm{~min} \text { of oral glucose } \\
\text { tolerence test (OGTT) }(\mathrm{mg} / \mathrm{dll})\end{array}$ & GLU60 & 1,135 & 133.307 & 37.708 & 268 & 249.451 & 52.941 & -33.940 & $<.0001$ \\
\hline Plasma glucose after $120 \mathrm{~min}$ of OGTT (mg/dL) & GLU120 & 1,138 & 101.917 & 21.315 & 268 & 247.705 & 61.951 & -38.000 & $<.0001$ \\
\hline Area under glucose curve $(\mathrm{mg} / \mathrm{dL} \cdot \mathrm{hr})$ & AUCGLU & 1,135 & 224.563 & 42.776 & 268 & 430.213 & 90.504 & -36.250 & $<.0001$ \\
\hline Fasting plasma insulin $(\mu \mathrm{U} / \mathrm{mL})$ & INSO & 1,138 & 7.459 & 6.754 & 328 & 8.879 & 6.918 & -3.340 & 0.0009 \\
\hline Plasma insulin after $60 \mathrm{~min}$ of OGTT ( $\mu \mathrm{U} / \mathrm{mL})$ & INS60 & 1,135 & 33.320 & 31.813 & 267 & 26.245 & 28.176 & 3.340 & 0.0009 \\
\hline Plasma insulin after $120 \mathrm{~min}$ of $\mathrm{OGTT}(\mu \mathrm{U} / \mathrm{mL})$ & INS120 & 1,138 & 23.436 & 24.317 & 267 & 33.386 & 40.686 & -3.840 & 0.0001 \\
\hline Area under insulin curve $(\mu \mathrm{U} / \mathrm{mL} \cdot \mathrm{hr})$ & AUCINS & 1,135 & 48.773 & 39.283 & 267 & 47.359 & 44.102 & 0.480 & 0.6308 \\
\hline Hemoglobin A1c (\%) & $\mathrm{HbA1c}$ & 1,138 & 5.486 & 0.241 & 483 & 7.355 & 1.534 & -26.640 & $<.0001$ \\
\hline Homeostasis model assessment of insulin resistance & HOMA-IR & 1,138 & 1.493 & 1.364 & 326 & 2.578 & 1.959 & -9.370 & $<.0001$ \\
\hline Microalbuminuria $(\mathrm{mg} / \mathrm{d})$ & MALB_U & 445 & 1.776 & 2.888 & 165 & 4.239 & 13.163 & -2.380 & 0.0183 \\
\hline
\end{tabular}

*Test statistics between normal and T2DM using student t-test for all variables except sex, whichwas compared by chi square test. 
pared by student t-test for all variables, except sex, which was compared by the chi square test. Deviation of genotype frequency from the expected Hardy-Weinberg equilibrium was examined with the chi square test. To approximate a normal distribution, TG, INS 0, INS60, INS120, AUCINS, HOMA-IR, QUICKI, ISI, and MALB_U were log-transformed before analysis. We examined linkage disequilibrium (D') and generated a plot for the PTPN1 gene using Haploview v3.2 (http://broad.mit.edu/ haploview/) (Barrett et al., 2005). Haplotypes were inferred using Haploview. Differences in genotype frequencies between T2DM patients and controls were compared using the chi square test, and the mode of inheritance was analyzed by a logistic regression procedure. Genotypes were given codes of 0,1 and 2; 0 , 1 and $1 ; 0,0$ and 1 in the additive, dominant, and recessive models, respectively. The associations between SNPs or haplotypes and T2DM-related subphenotypes were determined by linear regression analysis while controlling for age, sex, and BMI among normal control subjects. The SAS statistical software package (SAS Institute Inc. Cary, NC, USA) was used to perform general statistical analyses. Statistical significance was determined at a two-tailed value of $p<0.05$.

\section{Korean SNP database}

Information on most of the SNPs that are described in this study is available in the Korean SNP database (http://www.ngri,re.kr/SNP/), which was constructed at the Center for Genome Sciences (Korean National Institute of Health).

\section{Results}

Most of the variables that are shown in Table 1 were significantly higher in T2DM patients than normal controls, but HDL cholesterol and INS60 [insulin level at 1 hour after oral glucose tolerance test (OGTT)] were lower in T2DM patients than in normal controls, and no significant differences were observed in diastolic blood pressure or AUCINS (area under the curve in insulin level during OGTT) between groups.

Through direct sequencing of all exons and their boundaries in the PTPN1 gene, including up to -1500 bp of the 5' -flanking region, 19 SNPs were identified (Table 2). The genomic positions of the SNPs are illustrated in Fig. 1A. The SNPs that were selected for further study are in bold and with an asterisk (*); they had more than a $5 \%$ allele frequency and a tagging of the linkage disequilibrium (LD) block (data not shown).

None of the SNPS deviated from HWE (Hardy-

Table 2. Nineteen SNPs identified by direct sequencing in 24 unrelated subjects and one insertion/deletion (indel)

\begin{tabular}{|c|c|c|c|c|c|c|c|c|c|c|c|}
\hline Type & Position & $\begin{array}{l}\text { Position from } \\
\text { TSS in gene }\end{array}$ & $\begin{array}{l}\text { Position from } \\
\text { TSS in coding } \\
\text { region }\end{array}$ & $\begin{array}{l}\text { Major } \\
\text { Allele }\end{array}$ & $\begin{array}{l}\text { Minor } \\
\text { Allele }\end{array}$ & $\begin{array}{l}\text { Minor allele } \\
\text { Frequency }\end{array}$ & $\begin{array}{c}\text { Matching } \\
\text { with dbSNP }\end{array}$ & $\begin{array}{l}\text { Position } \\
\text { in exon }\end{array}$ & $\begin{array}{l}\text { Amino } \\
\text { acid } \\
\text { position }\end{array}$ & $\begin{array}{l}\text { Amino } \\
\text { acid-wild } \\
\text { type }\end{array}$ & $\begin{array}{l}\text { Amino acid- } \\
\text { mutant type }\end{array}$ \\
\hline SNP & Promoter & -1810 & & C & $\mathrm{T}$ & 0.022 & novel & & & & \\
\hline SNP & Promoter & -1769 & & $\mathrm{~T}$ & C & 0.022 & novel & & & & \\
\hline SNP & Intron 1 & 218 & & A & C & 0.295 & rs6067471 & & & & \\
\hline$\underline{\text { SNP }}$ & Intron 1 & $\underline{36,171}$ & & $\underline{G}$ & $\underline{A}$ & $\underline{0.354}$ & rs941798 & & & & \\
\hline SNP & Intron 3 & 54,646 & & G & T & 0.021 & novel & & & & \\
\hline$\underline{\mathrm{SNP}}$ & Intron 4 & $\underline{58,166}$ & & $\underline{G}$ & $\underline{A}$ & $\underline{0.063}$ & rs3787343 & & & & \\
\hline$\underline{\text { SNP }}$ & Intron 4 & $\underline{58,208}$ & & $\underline{A}$ & $\underline{\mathrm{G}}$ & $\underline{0.375}$ & rs2904270 & & & & \\
\hline SNP & Intron 4 & 58,359 & & $\mathrm{~T}$ & C & 0.375 & rs3787345 & & & & \\
\hline SNP & Intron 5 & 64,164 & & C & A & 0.391 & rs1885177 & & & & \\
\hline$\underline{\text { SNP }}$ & Intron 5 & $\underline{64,840}$ & & $\underline{\mathrm{C}}$ & $\underline{T}$ & $\underline{0.302}$ & rs754118 & & & & \\
\hline SNP & Intron 6 & 68,184 & & A & $\mathrm{G}$ & 0.409 & rs968701 & & & & \\
\hline SNP & Intron 7 & 69,103 & & G & A & 0.313 & rs2282147 & & & & \\
\hline SNP & Exon 8 & 69,220 & 909 & C & $\mathrm{T}$ & 0.130 & rs2282146 & cds & 303 & Pro (CCC) & Pro (CCT) \\
\hline SNP & Intron 8 & 69,535 & & T & C & 0.391 & rs718049 & & & & \\
\hline SNP & Intron 8 & 69,538 & & A & $\mathrm{G}$ & 0.391 & rs718053 & & & & \\
\hline$\underline{\text { SNP }}$ & Intron 8 & $\underline{69,560}$ & & $\underline{\mathrm{C}}$ & $\underline{\mathrm{G}}$ & $\underline{0.065}$ & rs6020612 & & & & \\
\hline$\underline{\text { SNP }}$ & Intron 8 & $\underline{69,866}$ & & $\underline{\mathrm{G}}$ & $\underline{A}$ & $\underline{0.356}$ & rs718050 & & & & \\
\hline$\underline{\text { SNP }}$ & Intron 8 & $\underline{69,934}$ & & $\underline{T}$ & $\underline{\mathrm{G}}$ & $\underline{0.433}$ & rs3787348 & & & & \\
\hline SNP & E9 & 70,909 & 1,260 & G & A & 0.022 & novel & cds & 420 & Thr (ACG) & Thr (ACA) \\
\hline Insdel & Exon 10 & 72,363 & 1,484 & G & & 0.000 & & 3' UTR & & & \\
\hline
\end{tabular}

TSS: Translation start site, Underline: selected SNPs for further genotyping in Ansan-Ansung cohort participants. 
(A)

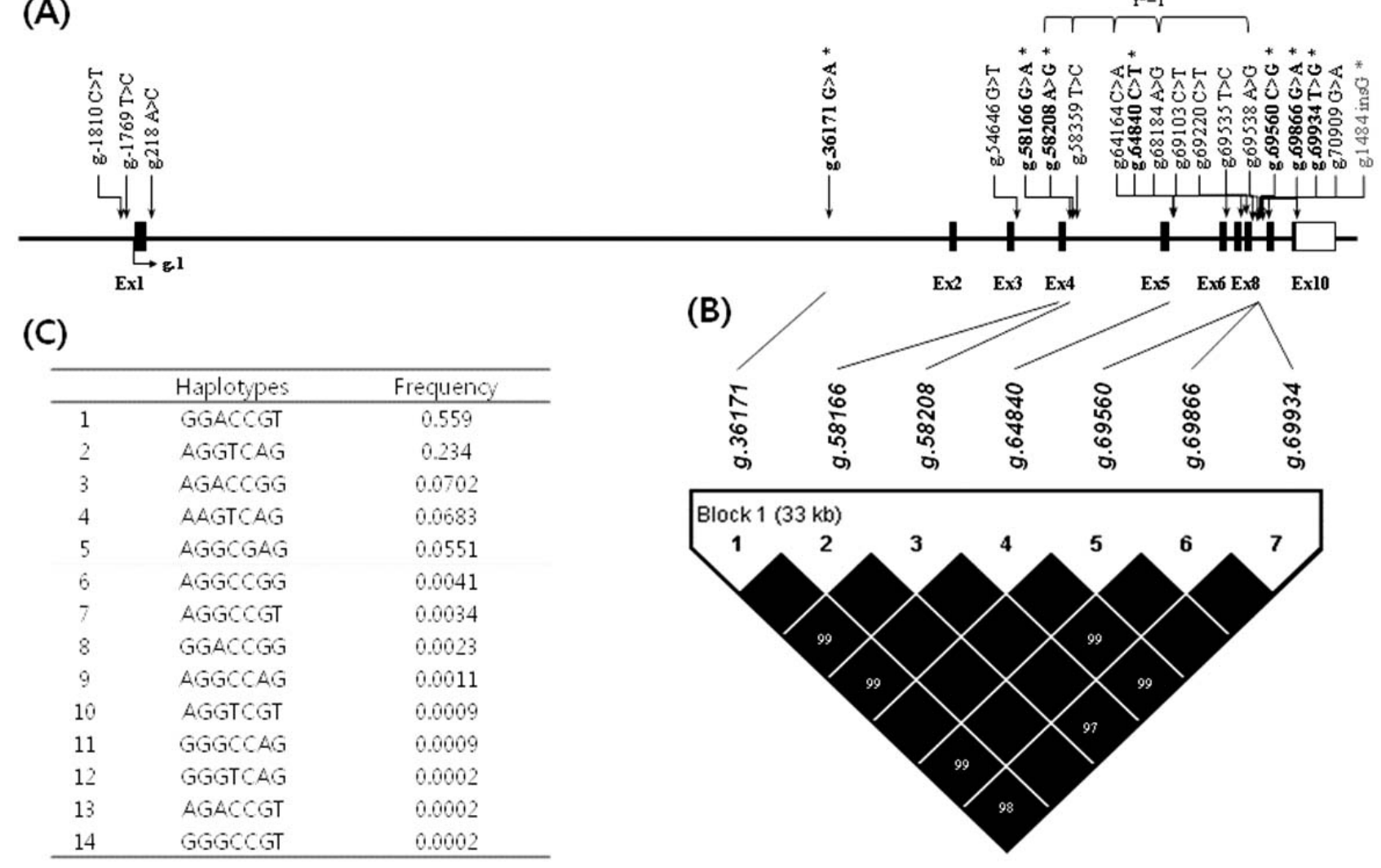

Fig. 1. (A) Map of PTPN1 (protein tyrosine phosphatase, non-receptor type 1) on chromosome 20q13.1-q13.2 (74 kb). Coding exons are marked by shaded blocks, and 5'- and 3'-untranslated regions are marked by white blocks. Asterisks $\left({ }^{*}\right)$ indicate SNPs that were genotyped in the larger population. The first nucleotide of the translational start site is denoted as 'nucleotide plus 1' (reference sequence of PTPN1: NT_011362). (B) Plot displaying linkage disequilibrium (LD) based on D' for the selected SNPs using the control population. All D' values were greater than 0.97. (C) Haplotype frequencies for seven SNPs selected from sequencing results and genotyped in normal control subjects.

Table 3. Logistic regression analysis for diabetic and the non-diabetic subjects with sex, age, and BMl adjustments

\begin{tabular}{|c|c|c|c|c|c|c|}
\hline & \multicolumn{2}{|l|}{ Codominant } & \multicolumn{2}{|l|}{ Dominant } & \multicolumn{2}{|l|}{ Recessive } \\
\hline & OR $(95 \% \mathrm{Cl})$ & $\mathrm{p}$ & OR $(95 \% \mathrm{Cl})$ & $\mathrm{p}$ & OR $(95 \% \mathrm{Cl})$ & $\mathrm{p}$ \\
\hline rs941798 (g.36171G >A) & $0.929(0.791 \sim 1.091)$ & 0.369 & $1.059(0.832 \sim 1.348)$ & 0.642 & $0.726(0.541 \sim 0.975)$ & $\underline{0.034}$ \\
\hline rs3787343 (g.58166G $>A)$ & $0.801(0.583 \sim 1.101)$ & 0.171 & $0.810(0.581 \sim 1.127)$ & 0.211 & $0.365(0.043 \sim 3.110)$ & 0.356 \\
\hline rs2904270 (g.58208A > G) & $0.991(0.842 \sim 1.167)$ & 0.916 & $1.071(0.854 \sim 1.344)$ & 0.553 & $0.838(0.602 \sim 1.165)$ & 0.293 \\
\hline rs754118 (g. 64840C >T) & $1.000(0.845 \sim 1.184)$ & 0.997 & $1.026(0.824 \sim 1.279)$ & 0.817 & $0.929(0.637 \sim 1.354)$ & 0.701 \\
\hline rs6020612 (g.69560C > G) & $0.771(0.535 \sim 1.113)$ & 0.165 & $0.761(0.521 \sim 1.111)$ & 0.157 & $0.846(0.086 \sim 8.326)$ & 0.886 \\
\hline rs718050 (g.69866G >A) & $0.970(0.823 \sim 1.143)$ & 0.718 & $1.036(0.826 \sim 1.298)$ & 0.762 & $0.817(0.582 \sim 1.147)$ & 0.244 \\
\hline rs3787348 (g.69934T > G) & $0.937(0.798 \sim 1.101)$ & 0.429 & $1.059(0.832 \sim 1.348)$ & 0.644 & $0.746(0.555 \sim 1.002)$ & 0.052 \\
\hline
\end{tabular}

Underline indicates significant result with $\mathrm{p}$-value $<0.05$.

Weinberg Equilibrium). An insertion/deletion variant (g.1484G_ins) was not polymorphic in our subjects. The selected seven SNPs belonged to an LD block with ID'| $>0.98$ (Fig. 1B). Five haplotypes with greater than a $5 \%$ frequency were predicted using the case-control sam- ples (Fig. 1C). The results of the logistic regression analysis are described in Table 3. rs941798 had a protective effect against diabetes, with an odds ratio of 0.726 (C.I. $0.541 \sim 0.975)$ and $p$-value $=0.034$. 
104 Genomics \& Informatics Vol. 6(3) 99-109, September 2008

Table 4. Linear regression analysis between PTPN1 genotypes and insulin resistance index adjusted for age, sex, and BMI

\begin{tabular}{|c|c|c|c|c|c|c|}
\hline & \multirow{2}{*}{ C (Major allele)/C } & \multirow{2}{*}{ C/R (Risk allele) } & \multirow{2}{*}{$\mathrm{R} / \mathrm{R}$} & \multirow{2}{*}{$\frac{\text { Codominant }}{p}$} & \multirow{2}{*}{$\frac{\text { Dominant }}{p}$} & \multirow{2}{*}{$\frac{\text { Recessive }}{p}$} \\
\hline & & & & & & \\
\hline \multicolumn{7}{|l|}{ rs941798 } \\
\hline GLU0 & $291(80.82 \pm 8.03)$ & $470(80.48 \pm 7.86)$ & $192(80.51 \pm 7.76)$ & 0.681 & 0.556 & 0.965 \\
\hline GLU60 & $290(132.29 \pm 37.03)$ & $468(133.67 \pm 40)$ & $192(132.7 \pm 38)$ & 0.671 & 0.593 & 0.895 \\
\hline GLU120 & $291(102.15 \pm 21.23)$ & $470(100.59 \pm 22.1)$ & $192(102.07 \pm 21.38)$ & 0.460 & 0.247 & 0.973 \\
\hline AUCGLU & $290(223.75 \pm 41.32)$ & $468(224.18 \pm 45.64)$ & $192(223.99 \pm 42.79)$ & 0.871 & 0.891 & 0.899 \\
\hline InINSO & $291(2.19 \pm 0.46)$ & $470(2.23 \pm 0.41)$ & $192(2.27 \pm 0.33)$ & 0.105 & 0.138 & 0.253 \\
\hline InINS60 & $290(3.19 \pm 0.86)$ & $468(3.24 \pm 0.8)$ & $192(3.21 \pm 0.8)$ & 0.802 & 0.545 & 0.798 \\
\hline InINS120 & $291(2.91 \pm 0.74)$ & $470(2.96 \pm 0.73)$ & $192(2.95 \pm 0.67)$ & 0.910 & 0.603 & 0.690 \\
\hline InAUCINS & $290(3.62 \pm 0.73)$ & $468(3.69 \pm 0.65)$ & $192(3.66 \pm 0.64)$ & 0.694 & 0.343 & 0.691 \\
\hline HBA1C & $291(5.48 \pm 0.24)$ & $470(5.49 \pm 0.23)$ & $192(5.49 \pm 0.22)$ & 0.635 & 0.587 & 0.835 \\
\hline InHOMAIR & $291(1.45 \pm 0.23)$ & $470(1.47 \pm 0.2)$ & $192(1.48 \pm 0.17)$ & 0.489 & 0.465 & 0.707 \\
\hline InQUICKI & $291(1.22 \pm 0.03)$ & $470(1.22 \pm 0.02)$ & $192(1.22 \pm 0.01)$ & 0.007 & $\underline{0.033}$ & 0.022 \\
\hline InISI & $290(1.39 \pm 0.25)$ & $468(1.36 \pm 0.17)$ & $192(1.36 \pm 0.19)$ & 0.114 & 0.029 & 0.788 \\
\hline \multicolumn{7}{|l|}{ rs3787343 } \\
\hline GLU0 & $812(80.73 \pm 8.05)$ & $132(79.7 \pm 6.52)$ & $6(85.67 \pm 10.88)$ & 0.645 & 0.385 & 0.093 \\
\hline GLU60 & $809(133.8 \pm 38.73)$ & $132(128.96 \pm 38.1)$ & $6(139.17 \pm 35.92)$ & 0.461 & 0.360 & 0.566 \\
\hline GLU120 & $812(101.69 \pm 21.49)$ & $132(99.16 \pm 22.96)$ & $6(111.67 \pm 18.66)$ & 0.224 & 0.136 & 0.383 \\
\hline AUCGLU & $809(224.99 \pm 43.67)$ & $132(218.39 \pm 43.53)$ & $6(237.83 \pm 41.65)$ & 0.327 & 0.215 & 0.384 \\
\hline InINSO & $812(2.22 \pm 0.41)$ & $132(2.23 \pm 0.4)$ & $6(2.31 \pm 0.09)$ & 0.991 & 0.960 & 0.863 \\
\hline InINS60 & $809(3.21 \pm 0.82)$ & $132(3.24 \pm 0.83)$ & $6(3.42 \pm 0.95)$ & 0.644 & 0.711 & 0.588 \\
\hline InINS120 & $812(2.94 \pm 0.73)$ & $132(2.93 \pm 0.69)$ & $6(2.64 \pm 0.72)$ & 0.301 & 0.428 & 0.170 \\
\hline InAUCINS & $809(3.66 \pm 0.68)$ & $132(3.68 \pm 0.68)$ & $6(3.73 \pm 0.77)$ & 0.952 & 0.959 & 0.954 \\
\hline HBA1C & $812(5.49 \pm 0.24)$ & $132(5.5 \pm 0.23)$ & $6(5.45 \pm 0.19)$ & 0.871 & 0.783 & 0.649 \\
\hline InHOMAIR & $812(1.47 \pm 0.21)$ & $132(1.47 \pm 0.2)$ & $6(1.5 \pm 0.03)$ & 0.764 & 0.730 & 0.911 \\
\hline InQUICKI & $812(1.22 \pm 0.02)$ & $132(1.22 \pm 0.02)$ & $6(1.21 \pm 0)$ & 0.610 & 0.682 & 0.549 \\
\hline InISI & $809(1.37 \pm 0.2)$ & $132(1.38 \pm 0.21)$ & $6(1.36 \pm 0.27)$ & 0.761 & 0.733 & 0.939 \\
\hline \multicolumn{7}{|l|}{ rs2904270 } \\
\hline GLU0 & $373(80.95 \pm 8.14)$ & $443(80.44 \pm 7.72)$ & $131(80.31 \pm 7.84)$ & 0.366 & 0.324 & 0.697 \\
\hline GLU60 & $372(132.67 \pm 37.88)$ & $441(133.78 \pm 40.58)$ & $131(132.54 \pm 34.77)$ & 0.715 & 0.629 & 0.971 \\
\hline GLU120 & $373(102.42 \pm 21.05)$ & $443(100.09 \pm 22.54)$ & $131(102.23 \pm 21.48)$ & 0.206 & 0.072 & 0.969 \\
\hline AUCGLU & $372(224.33 \pm 42.4)$ & $441(224.02 \pm 46.03)$ & $131(223.81 \pm 40.18)$ & 0.946 & 0.918 & 0.990 \\
\hline InINSO & $373(2.2 \pm 0.46)$ & $443(2.23 \pm 0.38)$ & $131(2.27 \pm 0.36)$ & 0.265 & 0.365 & 0.358 \\
\hline InINS60 & $372(3.19 \pm 0.84)$ & $441(3.24 \pm 0.79)$ & $131(3.24 \pm 0.85)$ & 0.585 & 0.537 & 0.837 \\
\hline InINS120 & $373(2.91 \pm 0.75)$ & $443(2.97 \pm 0.72)$ & $131(2.9 \pm 0.68)$ & 0.656 & 0.800 & 0.215 \\
\hline InAUCINS & $372(3.64 \pm 0.71)$ & $441(3.69 \pm 0.65)$ & $131(3.68 \pm 0.67)$ & 0.642 & 0.483 & 0.942 \\
\hline $\mathrm{HBA} 1 \mathrm{C}$ & $373(5.48 \pm 0.24)$ & $443(5.49 \pm 0.23)$ & $131(5.5 \pm 0.22)$ & 0.606 & 0.573 & 0.823 \\
\hline InHOMAIR & $373(1.46 \pm 0.23)$ & $443(1.46 \pm 0.18)$ & $131(1.48 \pm 0.19)$ & 0.819 & 0.942 & 0.728 \\
\hline InQUICKI & $373(1.22 \pm 0.03)$ & $443(1.22 \pm 0.02)$ & $131(1.22 \pm 0.01)$ & $\underline{0.013}$ & $\underline{0.023}$ & 0.087 \\
\hline InISI & $372(1.39 \pm 0.24)$ & $441(1.36 \pm 0.18)$ & $131(1.36 \pm 0.19)$ & $\overline{0.202}$ & $\overline{0.101}$ & 0.838 \\
\hline \multicolumn{7}{|l|}{ rs754118 } \\
\hline GLUO & $454(80.73 \pm 7.96)$ & $404(80.7 \pm 7.98)$ & $96(79.54 \pm 7.05)$ & 0.382 & 0.654 & 0.240 \\
\hline GLU60 & $453(131.71 \pm 37.37)$ & $402(134.98 \pm 41.33)$ & $96(130.72 \pm 33.06)$ & 0.461 & 0.264 & 0.813 \\
\hline GLU120 & $454(101.81 \pm 21.42)$ & $404(100.87 \pm 22.14)$ & $96(101.54 \pm 21.2)$ & 0.436 & 0.385 & 0.791 \\
\hline AUCGLU & $453(222.97 \pm 41.93)$ & $402(225.74 \pm 46.96)$ & $96(221.26 \pm 38.02)$ & 0.704 & 0.467 & 0.708 \\
\hline InINSO & $454(2.2 \pm 0.45)$ & $404(2.23 \pm 0.38)$ & $96(2.29 \pm 0.36)$ & 0.114 & 0.193 & 0.192 \\
\hline InINS60 & $453(3.21 \pm 0.84)$ & $402(3.21 \pm 0.8)$ & $96(3.27 \pm 0.79)$ & 0.645 & 0.856 & 0.479 \\
\hline InINS120 & $454(2.94 \pm 0.75)$ & $404(2.95 \pm 0.71)$ & $96(2.87 \pm 0.65)$ & 0.364 & 0.711 & 0.169 \\
\hline InAUCINS & $453(3.66 \pm 0.7)$ & $402(3.66 \pm 0.66)$ & $96(3.69 \pm 0.61)$ & 0.858 & 0.960 & 0.756 \\
\hline HBA1C & $454(5.49 \pm 0.24)$ & $404(5.49 \pm 0.23)$ & $96(5.47 \pm 0.24)$ & 0.540 & 0.822 & 0.330 \\
\hline InHOMAIR & $454(1.46 \pm 0.22)$ & $404(1.47 \pm 0.18)$ & $96(1.48 \pm 0.19)$ & 0.523 & 0.619 & 0.564 \\
\hline InQUICKI & $454(1.22 \pm 0.03)$ & $404(1.22 \pm 0.02)$ & $96(1.22 \pm 0.01)$ & $\underline{0.006}$ & $\underline{0.009}$ & 0.099 \\
\hline $\ln |S|$ & $453(1.38 \pm 0.23)$ & $402(1.37 \pm 0.19)$ & $96(1.35 \pm 0.16)$ & 0.213 & 0.282 & 0.344 \\
\hline
\end{tabular}


Table 4. Continued

\begin{tabular}{|c|c|c|c|c|c|c|}
\hline & \multirow{2}{*}{ C (Major allele)/C } & \multirow{2}{*}{ C/R (Risk allele) } & \multirow{2}{*}{$R / R$} & \multirow{2}{*}{$\frac{\text { Codominant }}{\mathrm{p}}$} & \multirow{2}{*}{$\frac{\text { Dominant }}{\mathrm{p}}$} & \multirow{2}{*}{$\frac{\text { Recessive }}{p}$} \\
\hline & & & & & & \\
\hline \multicolumn{7}{|l|}{ rs6020612 } \\
\hline GLUO & $854(80.55 \pm 7.87)$ & $99(80.98 \pm 7.78)$ & $2(74 \pm 11.31)$ & 0.916 & 0.776 & 0.239 \\
\hline GLU60 & $851(133.24 \pm 38.87)$ & $99(130.1 \pm 36.8)$ & $2(127 \pm 5.66)$ & 0.421 & 0.424 & 0.842 \\
\hline GLU120 & $854(101.43 \pm 21.68)$ & $99(100.64 \pm 22.74)$ & $2(99.5 \pm 24.75)$ & 0.361 & 0.354 & 0.918 \\
\hline AUCGLU & $851(224.21 \pm 43.96)$ & $99(220.91 \pm 41.81)$ & $2(213.75 \pm 23.69)$ & 0.359 & 0.369 & 0.759 \\
\hline InINSO & $854(2.22 \pm 0.41)$ & $99(2.24 \pm 0.39)$ & $2(2.1 \pm 0.42)$ & 0.775 & 0.816 & 0.682 \\
\hline InINS60 & $851(3.21 \pm 0.81)$ & $99(3.27 \pm 0.87)$ & $2(3.83 \pm 0.25)$ & 0.511 & 0.605 & 0.285 \\
\hline InINS120 & $854(2.93 \pm 0.72)$ & $99(3.04 \pm 0.75)$ & $2(3.5 \pm 0.54)$ & 0.215 & 0.268 & 0.254 \\
\hline InAUCINS & $851(3.65 \pm 0.67)$ & $99(3.74 \pm 0.7)$ & $2(4.18 \pm 0.01)$ & 0.326 & 0.398 & 0.259 \\
\hline HBA1C & $854(5.48 \pm 0.24)$ & $99(5.52 \pm 0.22)$ & $2(5.5 \pm 0.14)$ & 0.195 & 0.184 & 0.952 \\
\hline InHOMAIR & $854(1.46 \pm 0.21)$ & $99(1.47 \pm 0.18)$ & $2(1.39 \pm 0.2)$ & 0.797 & 0.849 & 0.614 \\
\hline InQUICKI & $854(1.22 \pm 0.02)$ & $99(1.22 \pm 0.02)$ & $2(1.22 \pm 0.02)$ & 0.929 & 0.947 & 0.865 \\
\hline InISI & $851(1.37 \pm 0.21)$ & $99(1.36 \pm 0.19)$ & $2(1.23 \pm 0.01)$ & 0.548 & 0.636 & 0.325 \\
\hline \multicolumn{7}{|l|}{ rs718050 } \\
\hline GLUO & $380(80.93 \pm 8.13)$ & $439(80.39 \pm 7.72)$ & $126(80.25 \pm 7.68)$ & 0.338 & 0.286 & 0.708 \\
\hline GLU60 & $379(132.93 \pm 37.95)$ & $437(133.35 \pm 40.48)$ & $126(131.75 \pm 34.11)$ & 0.922 & 0.853 & 0.942 \\
\hline GLU120 & $380(102.19 \pm 21.18)$ & $439(100.54 \pm 22.22)$ & $126(102.01 \pm 21.19)$ & 0.243 & 0.138 & 0.843 \\
\hline AUCGLU & $379(224.47 \pm 42.46)$ & $437(223.78 \pm 45.95)$ & $126(222.88 \pm 39.21)$ & 0.777 & 0.768 & 0.889 \\
\hline InINSO & $380(2.19 \pm 0.46)$ & $439(2.23 \pm 0.38)$ & $126(2.28 \pm 0.36)$ & 0.148 & 0.229 & 0.247 \\
\hline InINS60 & $379(3.19 \pm 0.84)$ & $437(3.23 \pm 0.79)$ & $126(3.26 \pm 0.84)$ & 0.523 & 0.549 & 0.680 \\
\hline InINS120 & $380(2.91 \pm 0.75)$ & $439(2.97 \pm 0.71)$ & $126(2.91 \pm 0.68)$ & 0.792 & 0.667 & 0.251 \\
\hline InAUCINS & $379(3.64 \pm 0.71)$ & $437(3.68 \pm 0.65)$ & $126(3.7 \pm 0.65)$ & 0.583 & 0.515 & 0.874 \\
\hline HBA1C & $380(5.48 \pm 0.24)$ & $439(5.49 \pm 0.23)$ & $126(5.49 \pm 0.23)$ & 0.817 & 0.729 & 0.969 \\
\hline InHOMAIR & $380(1.46 \pm 0.23)$ & $439(1.47 \pm 0.18)$ & $126(1.48 \pm 0.19)$ & 0.619 & 0.763 & 0.574 \\
\hline InQUICKI & $380(1.22 \pm 0.03)$ & $439(1.22 \pm 0.02)$ & $126(1.22 \pm 0.01)$ & 0.008 & 0.015 & 0.076 \\
\hline InISI & $379(1.38 \pm 0.23)$ & $437(1.36 \pm 0.18)$ & $126(1.36 \pm 0.17)$ & $\overline{0.160}$ & $\overline{0.118}$ & 0.578 \\
\hline \multicolumn{7}{|l|}{ rs3787348 } \\
\hline GLU0 & $295(80.92 \pm 8.03)$ & $467(80.5 \pm 7.85)$ & $191(80.42 \pm 7.76)$ & 0.518 & 0.437 & 0.809 \\
\hline GLU60 & $294(132.74 \pm 37.29)$ & $465(133.62 \pm 39.89)$ & $191(132.23 \pm 37.97)$ & 0.876 & 0.773 & 0.953 \\
\hline GLU120 & $295(102.1 \pm 21.33)$ & $467(100.81 \pm 22.02)$ & $191(101.85 \pm 21.49)$ & 0.441 & 0.292 & 0.889 \\
\hline AUCGLU & $294(224.23 \pm 41.51)$ & $465(224.25 \pm 45.56)$ & $191(223.37 \pm 42.69)$ & 0.917 & 0.943 & 0.920 \\
\hline InINSO & $295(2.18 \pm 0.45)$ & $467(2.23 \pm 0.41)$ & $191(2.27 \pm 0.33)$ & 0.071 & 0.092 & 0.218 \\
\hline InINS60 & $294(3.18 \pm 0.86)$ & $465(3.24 \pm 0.8)$ & $191(3.22 \pm 0.79)$ & 0.686 & 0.436 & 0.851 \\
\hline InINS120 & $295(2.9 \pm 0.74)$ & $467(2.96 \pm 0.73)$ & $191(2.95 \pm 0.67)$ & 0.789 & 0.481 & 0.732 \\
\hline InAUCINS & $294(3.62 \pm 0.72)$ & $465(3.69 \pm 0.66)$ & $191(3.66 \pm 0.63)$ & 0.598 & 0.281 & 0.752 \\
\hline HBA1C & $295(5.48 \pm 0.24)$ & $467(5.49 \pm 0.23)$ & $191(5.49 \pm 0.22)$ & 0.626 & 0.568 & 0.842 \\
\hline InHOMAIR & $295(1.45 \pm 0.22)$ & $467(1.47 \pm 0.2)$ & $191(1.48 \pm 0.17)$ & 0.415 & 0.382 & 0.670 \\
\hline InQUICKI & $295(1.22 \pm 0.03)$ & $467(1.22 \pm 0.02)$ & $191(1.22 \pm 0.01)$ & 0.006 & $\underline{0.026}$ & 0.021 \\
\hline InISI & $294(1.39 \pm 0.25)$ & $465(1.36 \pm 0.18)$ & $191(1.36 \pm 0.18)$ & $\overline{0.088}$ & $\overline{0.025}$ & $\overline{0.672}$ \\
\hline
\end{tabular}

Underline indicates significant result with $\mathrm{p}$-value $<0.05$.

The insulin resistance index had no significant association, except for log-transformed QUICKI and ISI (Table 4). The SNPs rs941798, rs2904270, rs9417114, rs718050, and rs3787348 showed significant association with QUICKI, but the differences were not distinguishable. The SNPs rs941798 and rs3787348 revealed a significant association with ISI, in which the individuals with minor alleles showed a decreased ISI.

Other quantitative traits that were related to T2DM were analyzed. Although there was no significant result between PTPN1 and the obesity indices (BMI and WHR) (data not shown), significant associations with blood pressure were observed for six SNPs (rs941798, rs2904270, rs754118, rs6020612, rs718050, and rs3787348) (Table 5). Individuals that had risk alleles of the six SNPs had significantly increased systolic and diastolic blood pressures. In addition, SNPs rs941798, rs2904270, rs754118, rs718050, and rs3787348 were associated with decreased HDL cholesterol levels in the codominant or dominant model (Table 6). SNP rs6020612 re- 
Table 5. Linear regression analysis between PTPN1 genotypes and blood pressure adjusted for age, sex, and BMI in nondiabetic subjects

\begin{tabular}{|c|c|c|c|c|c|c|}
\hline & \multirow{2}{*}{$\mathrm{C} / \mathrm{C}$} & \multirow{2}{*}{$\mathrm{C} / \mathrm{R}$} & \multirow{2}{*}{$\mathrm{R} / \mathrm{R}$} & \multirow{2}{*}{$\frac{\text { Codominant }}{\mathrm{p}}$} & \multirow{2}{*}{$\frac{\text { Dominant }}{p}$} & \multirow{2}{*}{$\begin{array}{c}\text { Recessive } \\
\mathrm{p}\end{array}$} \\
\hline & & & & & & \\
\hline \multicolumn{7}{|c|}{ rs941798 } \\
\hline SBP & $291(120.49 \pm 16)$ & $470(122.34 \pm 17.72)$ & $192(124.67 \pm 17.57)$ & 0.012 & 0.049 & 0.032 \\
\hline DBP & $291(75.15 \pm 9.45)$ & $470(76.09 \pm 9.65)$ & $192(78.23 \pm 9.87)$ & $\overline{0.001}$ & $\overline{0.032}$ & $\overline{0.002}$ \\
\hline \multicolumn{7}{|c|}{ rs3787343 } \\
\hline SBP & $812(122.17 \pm 17.05)$ & $132(122.89 \pm 18.59)$ & $6(114.78 \pm 11.5)$ & 0.901 & 0.888 & 0.225 \\
\hline DBP & $812(76.21 \pm 9.52)$ & $132(76.57 \pm 10.77)$ & $6(71.67 \pm 6.73)$ & 0.922 & 0.867 & 0.227 \\
\hline \multicolumn{7}{|c|}{ rs2904270 } \\
\hline SBP & $373(121.03 \pm 16.12)$ & $443(121.95 \pm 17.64)$ & $131(125.68 \pm 18.27)$ & $\underline{0.025}$ & 0.165 & $\underline{0.013}$ \\
\hline $\mathrm{DBP}$ & $373(75.44 \pm 9.4)$ & $443(76.1 \pm 9.5)$ & $131(78.42 \pm 10.77)$ & 0.008 & 0.090 & $\overline{0.005}$ \\
\hline \multicolumn{7}{|c|}{ rs754118 } \\
\hline SBP & $454(121.8 \pm 16.88)$ & $404(121.93 \pm 17.2)$ & $96(125.5 \pm 18.75)$ & 0.135 & 0.487 & 0.033 \\
\hline DBP & $454(75.69 \pm 9.48)$ & $404(76.45 \pm 9.73)$ & $96(77.84 \pm 10.26)$ & 0.042 & 0.116 & 0.065 \\
\hline \multicolumn{7}{|c|}{ rs6020612 } \\
\hline SBP & $854(121.72 \pm 17.1)$ & $99(126.27 \pm 17.6)$ & $2(139 \pm 26.87)$ & $\underline{0.017}$ & 0.025 & 0.141 \\
\hline $\mathrm{DBP}$ & $854(76.01 \pm 9.53)$ & $99(77.78 \pm 10.71)$ & $2(86.67 \pm 9.43)$ & $\overline{0.076}$ & $\overline{0.110}$ & 0.117 \\
\hline \multicolumn{7}{|c|}{ rs718050 } \\
\hline SBP & $380(121.11 \pm 16.56)$ & $439(121.92 \pm 17.39)$ & $126(126.47 \pm 18.34)$ & 0.014 & 0.168 & 0.003 \\
\hline DBP & $380(75.54 \pm 9.47)$ & $439(76.08 \pm 9.47)$ & $126(78.8 \pm 10.91)$ & $\overline{0.006}$ & 0.110 & $\overline{0.002}$ \\
\hline \multicolumn{7}{|c|}{ rs3787348 } \\
\hline SBP & $295(120.56 \pm 16.17)$ & $467(122.19 \pm 17.66)$ & $191(124.76 \pm 17.49)$ & 0.012 & 0.066 & 0.021 \\
\hline DBP & $295(75.27 \pm 9.42)$ & $467(76.02 \pm 9.71)$ & $191(78.16 \pm 9.81)$ & $\overline{0.003}$ & 0.059 & $\overline{0.002}$ \\
\hline
\end{tabular}

Underline indicates significant result with $\mathrm{p}$-value $<0.05$.

vealed increased triglycerides in the recessive model, but there were only 2 subjects that were homozygous for the minor allele.

\section{Discussion}

In this study, we investigated the effect of seven PTPN1 SNPs on susceptibility to T2DM and its related quantitative traits in a Korean Ansung-Ansan prospective community cohort. Our T2DM association results are similar to those that were found for the Caucasian-American population (Bento et al., 2004) and Hispanic-American population (Palmer et al., 2004) but differ from a European study (Florez et al., 2005), which did not find any significant associations even though they obtained the same haplotype block.

A total of five SNPs consisting of haplotype 2 (Fig. 1C) showed a significant association with QUICKI. Two (rs941798 and rs3787348) of the five SNPs also were associated with ISI. The results imply that the association between PTPN1 and T2DM may be caused by insulin resistance. Although the association with QUICKI was significant, the differences between genotypes were not clear. However, the ISI was decreased in individuals who had at least one minor allele. This result is well supported by a previous report (Palmer et al., 2004).

The association between the PTPN1 gene and blood pressure was greatly in concordance with previous two reports (Cheyssac et al., 2006; Spencer-Jones et al., 2005). Both reports suggested that PTPN1 gene variants increase blood pressure. Moreover, our results showed a more significant increase in systolic blood pressure in homozygotes of the SNP rs718050 minor allele. Although PTPN1 is an important regulator of the insulin signaling pathway, these results imply that SNP rs718050 may be a useful marker to predict hypertension.

We also identified the increased tendency of $T G$ in homozygotes of rs6020612; the minor allele homozygotes were observed in only two individuals. Although this association was reported in other reports (Cheyssac et al., 2006; Spencer-Jones et al., 2005), the associated SNP had a higher frequency of minor allele homozygotes. Therefore, the association between PTPN1 and TG should be confirmed using other SNPs that have been previously reported.

PTP1B also inhibits leptin signaling through the dephosphorylation of JAK2 and STAT3 (Zabolotny et al,, 2002; Cheng et al., 2002). Moreover, it was shown that inactivation with antisense oligonucleotides regulates the 
Table 6. Linear regression analysis between PTPN1 genotypes and lipidemic index adjusted for age, sex, and BMI in nondiabetic subjects

\begin{tabular}{|c|c|c|c|c|c|c|}
\hline & \multirow{2}{*}{$\mathrm{C} / \mathrm{C}$} & \multirow{2}{*}{$\mathrm{C} / \mathrm{R}$} & \multirow{2}{*}{$\mathrm{R} / \mathrm{R}$} & \multirow{2}{*}{$\frac{\text { Codominant }}{\mathrm{p}}$} & \multirow{2}{*}{$\frac{\text { Dominant }}{p}$} & \multirow{2}{*}{$\begin{array}{c}\text { Recessive } \\
\mathrm{p}\end{array}$} \\
\hline & & & & & & \\
\hline \multicolumn{7}{|l|}{ rs941798 } \\
\hline $\operatorname{lnTG}$ & $291(4.86 \pm 0.41)$ & $470(4.92 \pm 0.41)$ & $192(4.92 \pm 0.44)$ & 0.187 & 0.080 & 0.757 \\
\hline $\mathrm{TCHL}$ & $291(185 \pm 33.88)$ & $470(183.84 \pm 33.45)$ & $192(183.52 \pm 32.69)$ & 0.293 & 0.348 & 0.443 \\
\hline $\mathrm{HDL}$ & $291(47.12 \pm 11.04)$ & $470(45.09 \pm 9.57)$ & $192(44.16 \pm 9.51)$ & $\underline{0.002}$ & $\underline{0.002}$ & 0.064 \\
\hline LDL & $290(110.44 \pm 32.27)$ & $465(109.73 \pm 31.03)$ & $189(110.41 \pm 29.44)$ & 0.537 & 0.515 & 0.737 \\
\hline \multicolumn{7}{|c|}{ rs3787343 } \\
\hline $\operatorname{lnTG}$ & $812(4.9 \pm 0.42)$ & $132(4.89 \pm 0.4)$ & $6(4.78 \pm 0.55)$ & 0.621 & 0.714 & 0.476 \\
\hline TCHL & $812(183.55 \pm 33.45)$ & $132(188.62 \pm 32.93)$ & $6(172.51 \pm 40.73)$ & 0.474 & 0.303 & 0.233 \\
\hline $\mathrm{HDL}$ & $812(45.55 \pm 10.23)$ & $132(45.51 \pm 9.63)$ & $6(48.14 \pm 3.09)$ & 0.728 & 0.837 & 0.464 \\
\hline LDL & $803(109.45 \pm 31.01)$ & $132(114.73 \pm 30.67)$ & $6(98.09 \pm 49.65)$ & 0.440 & 0.260 & 0.179 \\
\hline \multicolumn{7}{|c|}{ rs2904270 } \\
\hline $\operatorname{lnTG}$ & $373(4.87 \pm 0.39)$ & $443(4.93 \pm 0.42)$ & $131(4.92 \pm 0.45)$ & 0.107 & 0.052 & 0.662 \\
\hline TCHL & $373(183.53 \pm 33.69)$ & $443(184.61 \pm 33.07)$ & $131(185.5 \pm 34.47)$ & 0.937 & 0.898 & 0.979 \\
\hline HDL & $373(46.41 \pm 10.56)$ & $443(45.25 \pm 9.64)$ & $131(44.14 \pm 10.09)$ & 0.038 & 0.066 & 0.136 \\
\hline LDL & $372(109.72 \pm 32.12)$ & $438(110.17 \pm 30.45)$ & $129(111.81 \pm 30.71)$ & 0.995 & 0.925 & 0.885 \\
\hline \multicolumn{7}{|l|}{ rs754118 } \\
\hline InTG & $454(4.88 \pm 0.4)$ & $404(4.91 \pm 0.42)$ & $96(4.95 \pm 0.43)$ & 0.139 & 0.208 & 0.247 \\
\hline $\mathrm{TCHL}$ & $454(184.21 \pm 33.04)$ & $404(184.05 \pm 33.68)$ & $96(184.65 \pm 34.5)$ & 0.763 & 0.770 & 0.860 \\
\hline HDL & $454(46.16 \pm 10.51)$ & $404(45.3 \pm 9.62)$ & $96(43.8 \pm 9.91)$ & 0.049 & 0.113 & 0.090 \\
\hline LDL & $451(110.06 \pm 31.21)$ & $400(110 \pm 31.34)$ & $94(110.85 \pm 29.86)$ & 0.793 & 0.797 & 0.883 \\
\hline \multicolumn{7}{|c|}{ rs6020612 } \\
\hline $\operatorname{lnTG}$ & $854(4.9 \pm 0.41)$ & $99(4.91 \pm 0.46)$ & $2(5.67 \pm 0.07)$ & 0.644 & 0.939 & $\underline{0.007}$ \\
\hline TCHL & $854(184.06 \pm 33.54)$ & $99(185.93 \pm 32.65)$ & $2(184.35 \pm 2.63)$ & 0.979 & 0.980 & $\overline{0.986}$ \\
\hline HDL & $854(45.65 \pm 10.04)$ & $99(44.76 \pm 10.33)$ & $2(32.68 \pm 1.71)$ & 0.321 & 0.453 & 0.069 \\
\hline LDL & $848(109.93 \pm 31.26)$ & $96(112.73 \pm 29.65)$ & $2(93.94 \pm 4.92)$ & 0.889 & 0.797 & 0.454 \\
\hline \multicolumn{7}{|l|}{ rs718050 } \\
\hline InTG & $380(4.87 \pm 0.4)$ & $439(4.91 \pm 0.42)$ & $126(4.95 \pm 0.45)$ & 0.082 & 0.130 & 0.196 \\
\hline $\mathrm{TCHL}$ & $380(183.65 \pm 33.82)$ & $439(184.49 \pm 32.95)$ & $126(184.85 \pm 34.39)$ & 0.847 & 0.941 & 0.779 \\
\hline $\mathrm{HDL}$ & $380(46.34 \pm 10.53)$ & $439(45.38 \pm 9.67)$ & $126(43.43 \pm 9.96)$ & $\underline{0.016}$ & 0.073 & $\underline{0.027}$ \\
\hline LDL & $379(109.62 \pm 32.25)$ & $434(110.3 \pm 30.23)$ & $123(111.46 \pm 30.65)$ & $\overline{0.931}$ & 0.938 & $\overline{0.950}$ \\
\hline \multicolumn{7}{|c|}{ rs3787348 } \\
\hline InTG & $295(4.87 \pm 0.41)$ & $467(4.91 \pm 0.41)$ & $191(4.92 \pm 0.44)$ & 0.254 & 0.207 & 0.579 \\
\hline $\mathrm{TCHL}$ & $295(185.11 \pm 34.03)$ & $467(184.09 \pm 33.59)$ & $191(183.1 \pm 32.3)$ & 0.241 & 0.345 & 0.330 \\
\hline HDL & $295(47.07 \pm 11.08)$ & $467(45.18 \pm 9.54)$ & $191(44.07 \pm 9.55)$ & $\underline{0,002}$ & $\underline{0.003}$ & $\underline{0.044}$ \\
\hline LDL & $294(110.29 \pm 32.51)$ & $462(110.19 \pm 31.08)$ & $188(109.87 \pm 29.1)$ & 0.503 & 0.632 & 0.531 \\
\hline
\end{tabular}

Underline indicates significant result with $p$-value $<0.05$.

expression of genes that are involved in lipogenesis, such as SREBF1, suggesting that PTP1B may play a role in the enlargement of adipocyte energy storage (Rondinone et al., 2002). Two SNPs (rs941798 and rs3787348) effected decreased HDL cholesterol levels in minor allele homozygotes. This result also has been replicated in another report (Cheyssac et al., 2006), but the previous report showed a marginal association, while our results revealed lower p-values (rs941794 $p=0.002$, and $r s 3787348 p=0.003$ ). Both SNPs showed a significant association with blood pressure, implying that the increased blood pressure might result in low HDL cholesterol levels and be related to lipid metabolism, which is another molecular pathway of PTPN1 function (Santaniemi et al., 2004).

This is the first report that shows the association between PTPN1 and T2DM in the Korean as well as Asian population. We hope this study will increase our knowledge about T2DM pathophysiology.

\section{Acknowledgments}

This study was supported by an intramural grant from the National Institute of Health, Korea and an intramural grant from Kyung Hee University. 


\section{References}

Benchabane, H., and Wrana, J.L. (2003). GATA- and Smad1-dependent enhancers in the Smad7 gene differentially interpret bone morphogenetic protein concentrations. Mol. Cell. Biol. 23, 6646-6661.

Bento, J.L., Palmer, N.D., Mychaleckyj, J.C., Lange, L.A., Langefeld, C.D., Rich, S.S., Freedman, B.I., and Bowden, D.W. (2004). Association of protein tyrosine phosphatase 1B gene polymorphisms with type 2 diabetes. Diabetes 53, 3007-3012.

Bowden, D.W., Sale, M., Howard, T.D., Qadri, A., Spray, B.J., Rothschild, C.B., Akots, G., Rich, S.S., and Freedman, B.I. (1997). Linkage of genetic markers on human chromosomes 20 and 12 to NIDDM in Caucasian sib pairs with a history of diabetic nephropathy. Diabetes 46, 882-886.

Burdon, K.P., Bento, J.L., Langefeld, C.D., Campbell, J.K., Carr, J.J., Wagenknecht, L.M., Herrington, D.M., Freedman, B.I., Rich, S.S., and Bowden, D.W. (2006). Association of protein tyrosine phosphatase-N1 polymorphisms with coronary calcified plaque in the Diabetes Heart Study. Diabetes 55, 651-658.

Cheng, A., Uetani, N., Simoncic, P.D., Chaubey, V.P., LeeLoy, A., McGlade, C.J., Kennedy, B.P., and Tremblay, M.L. (2002). Attenuation of leptin action and regulation of obesity by protein tyrosine phosphatase 1B. Dev. Cell 2, 497-503.

Cheyssac, C., Lecoeur, C., Dechaume, A., Bibi, A., Charpentier, G., Balkau, B., Marre, M., Froguel, P., Gibson, F., and Vaxillaire, M. (2006). Analysis of common PTPN1 gene variants in type 2 diabetes, obesity and associated phenotypes in the French population. BMC Med. Genet. $7,44$.

Collaku, A., Rankinen, T., Rice, T., Leon, A.S., Rao, D.C., Skinner, J.S., Wilmore, J.H., and Bouchard, C. (2004). A genome-wide linkage scan for dietary energy and nutrient intakes: the Health, Risk Factors, Exercise Training, and Genetics (HERITAGE) Family Study. Am. J. Clin. Nutr. 79, 881-886.

Di Paola, R., Frittitta, L., Miscio, G., Bozzali, M., Baratta, R., Centra, M., Spampinato, D., Santagati, M.G., Ercolino, T., Cisternino, C., Soccio, T., Mastroianno, S., Tassi, V., Almgren, P., Pizzuti, A., Vigneri, R., and Trischitta, V. (2002). A variation in $3^{\prime}$ UTR of hPTP1B increases specific gene expression and associates with insulin resistance. Am. J. Hum. Genet. 70, 806-812.

Dong, C., Wang, S., Li, W.D., Li, D., Zhao, H., and Price, R.A. (2003). Interacting genetic loci on chromosomes 20 and 10 influence extreme human obesity. Am. J. Hum. Genet. 72, 115-124.

Eberle, M. A., and Kruglyak, L. (2000). An analysis of strategies for discovery of single-nucleotide polymorphisms. Genet. Epidemiol. 19, Suppl. 1, S29-35.

Echwald, S.M., Bach, H., Vestergaard, H., Richelsen, B., Kristensen, K., Drivsholm, T., Borch-Johnsen, K., Hansen, T., and Pedersen, O. (2002). A P387L variant in protein tyrosine phosphatase-1B (PTP-1B) is associated with type 2 diabetes and impaired serine phosphorylation of
PTP-1B in vitro. Diabetes 51, 1-6.

Elchebly, M., Payette, P., Michaliszyn, E., Cromlish, W., Collins, S., Loy, A.L., Normandin, D., Cheng, A., HimmsHagen, J., Chan, C.C., Ramachandran, C., Gresser, M.J., Tremblay, M.L., and Kennedy, B.P. (1999). Increased insulin sensitivity and obesity resistance in mice lacking the protein tyrosine phosphatase-1B gene. Science 283, 1544-1548.

Evans, J.L., and Jallal, B. (1999). Protein tyrosine phosphatases: their role in insulin action and potential as drug targets. Expert Opin. Investig. Drugs. 8, 139-160.

Florez, J.C., Agapakis, C.M., Burtt, N.P., Sun, M., Almgren, P., Rastam, L., Tuomi, T., Gaudet, D., Hudson, T.J., Daly, M.J., Ardlie, K.G., Hirschhorn, J.N., Groop, L., and Altshuler, D. (2005). Association testing of the protein tyrosine phosphatase 1B gene (PTPN1) with type 2 diabetes in 7,883 people. Diabetes 54, 1884-1891.

Forsell, P.A., Boie, Y., Montalibet, J., Collins, S., and Kennedy, B.P. (2000). Genomic characterization of the human and mouse protein tyrosine phosphatase-1B genes. Gene 260, 145-153.

Goldstein, B.J., Bittner-Kowalczyk, A., White, M.F., and Harbeck, M. (2000). Tyrosine dephosphorylation and deactivation of insulin receptor substrate-1 by protein-tyrosine phosphatase 1B. Possible facilitation by the formation of a ternary complex with the Grb2 adaptor protein. J. Biol. Chem. 275, 4283-4289.

Goldstein, B.J., Li, P.M., Ding, W., Ahmad, F., and Zhang, W.R. (1998). Regulation of insulin action by protein tyrosine phosphatases. Vitam. Horm. 54, 67-96.

Hunt, S.C., Abkevich, V., Hensel, C.H., Gutin, A., Neff, C.D.,

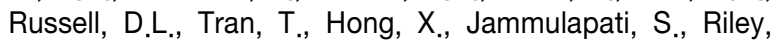
R., Weaver-Feldhaus, J., Macalma, T., Richards, M.M., Gress, R., Francis, M., Thomas, A., Frech, G.C., Adams, T.D., Shattuck, D., and Stone, S. (2001). Linkage of body mass index to chromosome 20 in Utah pedigrees. Hum. Genet. 109, 279-285.

Ji, L., Malecki, M., Warram, J.H., Yang, Y., Rich, S.S., and Krolewski, A.S. (1997). New susceptibility locus for NIDDM is localized to human chromosome 20q. Diabetes 46, 876-881.

Katz, A., Nambi, S.S., Mather, K., Baron, A.D., Follmann, D.A., Sullivan, G., and Quon M.J. (2000). Quantitative insulin sensitivity check index: a simple, accurate method for assessing insulin sensitivity in humans. J. Clin. Endocrinol. Metab. 85, 2402-2410.

Klaman, L.D., Boss, O., Peroni, O.D., Kim, J.K., Martino, J.L., Zabolotny, J.M., Moghal, N., Lubkin, M., Kim, Y.B., Sharpe, A.H., Stricker-Krongrad, A., Shulman, G.I., Neel, B.G., and Kahn, B.B. (2000). Increased energy expenditure, decreased adiposity, and tissue-specific insulin sensitivity in protein-tyrosine phosphatase 1B-deficient mice. Mol. Cell Biol. 20, 5479-5489.

Klupa, T., Malecki, M.T., Pezzolesi, M., Ji, L., Curtis, S., Langefeld, C.D., Rich, S.S., Warram, J.H., and Krolewski, A.S. (2000). Further evidence for a susceptibility locus for type 2 diabetes on chromosome 20q13.1-q13.2. Diabetes 49, 2212-2216

Kwan, M., Powell, D.R., Nachman, T.Y., and Brown, M.A. 
(2005). An intron GATA-binding site regulates chromatin accessibility and is essential for IL-4 gene expression in mast cells. Eur. J. Immunol. 35, 1267-1274.

Lembertas, A.V., Perusse, L., Chagnon, Y.C., Fisler, J.S., Warden, C.H., Purcell-Huynh, D.A., Dionne, F.T., Gagnon, J., Nadeau, A., Lusis, A.J., and Bouchard, C. (1997). Identification of an obesity quantitative trait locus on mouse chromosome 2 and evidence of linkage to body fat and insulin on the human homologous region $20 \mathrm{q}$. J. Clin. Invest. 100, 1240-1247.

Livak, K.J. (1999). Allelic discrimination using Xuorogenic probes and the 5_nuclease assay. Genet. Anal. 14, 143-149

Palmer, N.D., Bento, J.L., Mychaleckyj, J.C., Langefeld, C.D., Campbell, J.K., Norris, J.M., Haffner, S.M., Bergman, R.N., and Bowden, D.W. (2004). Association of protein tyrosine phosphatase $1 \mathrm{~B}$ gene polymorphisms with measures of glucose homeostasis in Hispanic Americans: the insulin resistance atherosclerosis study (IRAS) family study. Diabetes 53, 3013-3019.

Rondinone, C.M., Trevillyan, J.M., Clampit, J., Gum, R. J., Berg, C., Kroeger, P., Frost, L., Zinker, B.A., Reilly, R., Ulrich, R., Butler, M., Monia, B.P., Jirousek, M.R., and Waring, J.F. (2002). Protein tyrosine phosphatase 1B reduction regulates adiposity and expression of genes involved in lipogenesis. Diabetes 51, 2405-2411.
Santaniemi, M., Ukkola, O., and Kesaniemi, Y.A. (2004). Tyrosine phosphatase $1 \mathrm{~B}$ and leptin receptor genes and their interaction in type 2 diabetes. J. Intern. Med. 256, 48-55.

Seely, B.L., Staubs, P.A., Reichart, D.R., Berhanu, P., Milarski, K.L., Saltiel, A.R., Kusari, J., and Olefsky, J.M. (1996). Protein tyrosine phosphatase 1B interacts with the activated insulin receptor. Diabetes 45, 1379-1385.

Spencer-Jones, N.J., Wang, X., Snieder, H., Spector, T.D., Carter, N.D., and O'Dell, S.D. (2005). Protein tyrosine phosphatase-1B gene PTPN1: selection of tagging single nucleotide polymorphisms and association with body fat, insulin sensitivity, and the metabolic syndrome in a normal female population. Diabetes 54, 3296-3304.

Zabolotny, J.M., Bence-Hanulec, K.K., Stricker-Krongrad, A., Haj, F., Wang, Y., Minokoshi, Y., Kim, Y.B., Elmquist, J.K., Tartaglia, L.A., Kahn, B.B., and Neel, B.G. (2002). PTP1B regulates leptin signal transduction in vivo. Dev. Cell 2, 489-495.

Zouali, H., Hani, E.H., Philippi, A., Vionnet, N., Beckmann, J.S., Demenais, F., and Froguel, P. (1997). A susceptibility locus for early-onset non-insulin dependent (type 2) diabetes mellitus maps to chromosome $20 \mathrm{q}$, proximal to the phosphoenolpyruvate carboxykinase gene. Hum. Mol. Genet. 6, 1401-1408. 